\title{
Relation nappe-rivière dans le bassin versant du Bandama en milieu de socle fissuré en Côte d'Ivoire : approche couplée hydrochimie - télédétection
}

\author{
Bamory KAMAGATE * , Droh Lanciné GONE, Inza DOUMOUYA, \\ Ismaïla OUATTARA, Moussa OUEDRAOGO, Adama BAMBA et Issiaka SAVANE \\ Laboratoire de Géosciences et Environnement, UFR des Sciences et gestion de l'Environnement, Université \\ d'Abobo-Adjamé, 02 BP 801 Abidjan 02, Côte d'Ivoire. \\ *Auteur correspondant, E-mail: kambamory2@yahoo.fr, Tél : + (225) 44861810
}

\section{RESUME}

Le bassin versant du fleuve Bandama a été retenu pour mieux comprendre les interactions entre le réseau hydrographique et les eaux souterraines des aquifères d'altérites et du socle fissuré. A partir d'analyses physico-chimiques $\left(\mathrm{T}^{\circ}, \mathrm{pH}\right.$, conductivité électrique, cations et anions majeurs) portant sur des échantillons d'eau prélevés à la fois dans les cours d'eau, les nappes d'altérites (puits) et les nappes de socle (forage), la signature chimique de chaque système hydrique a été établie. En appui à l'hydrochimie, le traitement d'images satellitaires a permis de ressortir le réseau hydrographique et le réseau des fractures du socle sous-jacent. Les eaux de surface présentent une signature chimique proche de celles des aquifères du socle fissuré avec une bonne superposition entre le réseau des cours d'eau et le réseau des fractures du socle cristallin. Ceci traduit l'existence de conditions favorables aux échanges de flux entre le réseau hydrographique et les aquifères fissurés, sans contribution significative des aquifères d'altérites. Les fractures étant en contact avec le réseau hydrographique, celles-ci pourraient jouer un rôle de drain pour les cours d'eau du bassin.

(c) 2011 International Formulae Group. All rights reserved.

Mots clés : Eaux de surface, aquifères d'altérites, aquifères de socle, hydrochimie, bassin du Bandama, Côte d'Ivoire.

\section{INTRODUCTION}

Le bassin versant du Bandama a fait l'objet de nombreuses études portant sur les changements hydroclimatiques des années 1970 et leur impact sur les hydrosystèmes (Grillot, 1992 ; Servat et al., 1997 ; Lebel et Vischel, 2005 ; Goula et al., 2006). Ces changements se traduisent par une baisse pluviométrique de l'ordre de $10 \%$. Le déficit des écoulements associé à cette baisse s'élève à près de $50 \%$ attestant d'une absence de linéarité entre les précipitations et les débits des cours d'eau (Doumouya et al., 2009).
Cette relation non linéaire entre pluie et débit, ainsi que sa variabilité spatio-temporelle, sont encore mal comprises à l'échelle de ce bassin. Les principales études réalisées ont été menées à partir d'approches indirectes ou conceptuelles (tests statistiques ou calculs de coefficients de tarissement ; Goula et al., 2006 ; Ouédraogo, 2009 ; Ouattara, 2009).

Les cours d'eau étant des exhaures naturelles de différents réservoirs hydriques (eaux de surface et eaux souterraines) et jouant un rôle essentiel dans l'équilibre hydrodynamique de l'ensemble du bassin versant (Braun et al., 
2002), une explication possible de la diminution relativement plus importante des débits des cours d'eau serait la réduction durable des apports en eaux souterraines (Kamagaté, 2006 ; Dao et al., 2010). La validation de cette hypothèse nécessiterait une analyse des processus physiques à une échelle comparable à celle des observations de surface (disponibilité de données piézométriques sur une période décennale ou pluridécennale identique à celle des observations de débits). Toutefois, des éléments de réponse peuvent être apportés à partir de la caractérisation des processus physiques impliquant les différents réservoirs hydriques d'un bassin versant représentatif, portant en particulier sur les relations napperivière. L'objectif de ce travail est d'appréhender la relation entre le réseau hydrographique et les eaux souterraines. Il s'agit de mieux comprendre le type de relation entre les cours d'eau du bassin versant du Bandama et les nappes sous-jacentes en vue de mieux apprécier la part de chaque compartiment dans la production des débits des cours d'eau.

\section{MATERIEL ET METHODES Milieu d'étude}

Le bassin du Bandama est situé entre les longitudes $3^{\circ} 50^{\prime}$ et $7^{\circ} \mathrm{W}$, et entre les latitudes $5^{\circ} 10^{\prime}$ et $10^{\circ} 20^{\prime} \mathrm{N}$. Ce bassin, d'une superficie de $97500 \mathrm{~km}^{2}$, s'étand du nord au sud de la Côte d'Ivoire (Figure 1). Il est constitué au plan géomorphologique de pénéplaines dont l'altitude varie respectivement de 250 à $350 \mathrm{~m}$ au nord et de 50 à $100 \mathrm{~m}$ au sud. Le régime pluviométrique est de type tropical de transition dans la partie septentrionale, équatorial de transition atténué dans la partie centrale et équatorial de transition dans la partie méridionale (Servat et al., 1997). L'analyse de la pluviométrie et de la température à la station écologique de Lamto (1964-2004) révèle que les moyennes mensuelles sont respectivement de $1200 \mathrm{~mm}$ et $28^{\circ} \mathrm{C}$.
La couverture pédologique de type ferrugineux tropical recouvre une zone d'altération à épaisseur variable (50 m par endroit) et à nappe phréatique. Cette zone est principalement constituée de formation argileuses, silteuses, sableuses et arénitiques (Monnet, 1970 ; Kouamé et al., 2008). Sousjacent à ce recouvrement altéritique, le substratum cristallin et cristallophyllien laisse apparaître localement des fractures qui peuvent constituer des réservoirs d'eaux souterraines. Ce socle est essentiellement constitué de formations granito-gneissiques et migmatitiques (Jourda, 2002).

\section{Approche hydrochimique}

Les données hydrochimiques, obtenues après une campagne de mesures, sont constituées de mesures physico-chimiques (température, $\mathrm{pH}$ et conductivité électrique) réalisées in situ au moyen de multimètre WTW et des prélèvements d'eau conservés sur le terrain à $5{ }^{\circ} \mathrm{C}$ dans une glacière et destinés à l'analyse d'ions majeurs au laboratoire. Ces analyses portent sur les échantillons d'eaux de 14 puits réalisés dans les aquifères d'altérites, 36 forages captant les aquifères de fissures et 8 échantillons d'eau de surface répartis sur l'ensemble de la zone d'étude.

Les méthodes analytiques varient selon les éléments chimiques recherchés; $\mathrm{Ca}^{2+}$, $\mathrm{Mg}^{2+}, \mathrm{Cl}^{-}$et $\mathrm{HCO}_{3}^{-}$par titrimétrie (titration acide), $\mathrm{Na}^{+}, \mathrm{K}^{+}$par spectrométrie d'absorption atomique à la flamme, $\mathrm{NH}_{4}{ }^{+}, \mathrm{PO}_{4}{ }^{3-}, \mathrm{SO}_{4}{ }^{2-}$, $\mathrm{NO}_{3}{ }^{-}, \quad \mathrm{NO}_{2}^{-}$et $\mathrm{SiO}_{2}$ par spectrométrie d'absorption moléculaire. Ces analyses ont été réalisées au laboratoire de physico-chimie du Centre Ivoirien Antipollution (CIAPOL).

Outre l'observation des concentrations des différents éléments chimiques analysés, un diagramme de Piper est réalisé pour caractériser les différents hydrofaciès. Le diagramme de Piper est une représentation des anions et des cations sur deux triangles spécifiques dont les côtés témoignent des teneurs relatives en chacun des ions majeurs par rapport au total de ces ions. 


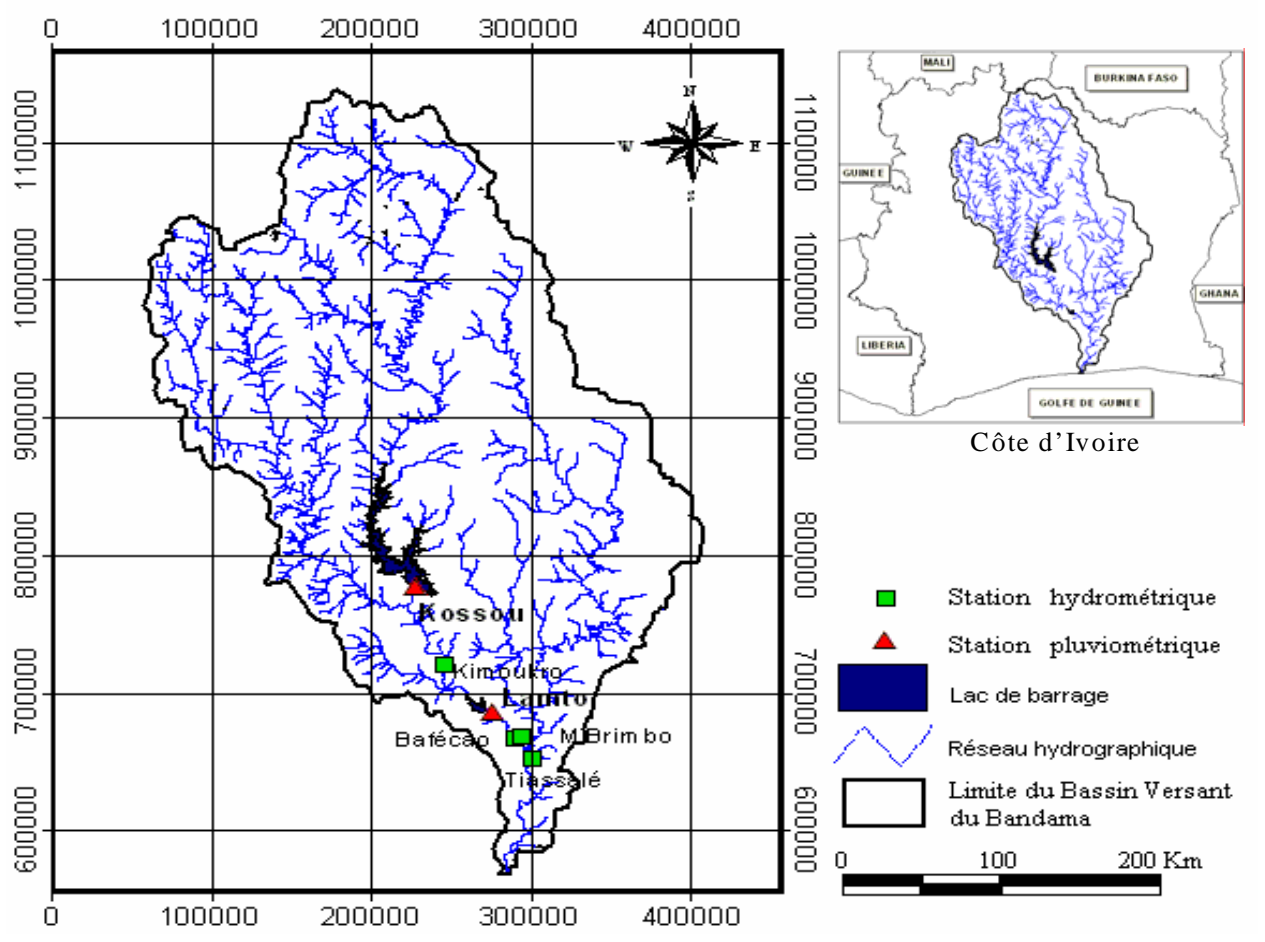

Figure 1 : Présentation de la zone d'étude.

\section{Approche par télédétection \\ Acquisition des données images}

La zone d'étude est couverte par les scènes d'images satellitaires 196/55; 196/56; 197/55; 197/55 TM de Landsat, enregistrées le 16 Janvier 1986 en l'absence de couverture nuageuse, période favorable à une bonne visibilité des capteurs. A ces images s'ajoute une carte topographique. Les logiciels ENVI 4.3, MapInfo 6.5 et Arcview 3.3 ont servi, respectivement, au traitement des images satellitaires et du réseau hydrographique, et au croisement des informations géoréférencées pour la mise en place du Système d’Information Géographique (SIG).

\section{Traitement des images}

L'objectif ici est de cartographier le réseau de linéaments (fractures probables) du socle afin d'étudier la relation entre les réseaux de fractures et hydrographique.

Les différentes images ont subi un prétraitement portant sur le géoréférencement et la mosaïque. Ensuite, l'analyse en composantes principales de bandes spectrales a été effectuée pour extraire les informations de l'ensemble des canaux bruts. Cela permet de prendre en compte toute l'information contenue dans chaque canal brut par la considération de la notion d'information en imagerie optique qui reste liée à la variance des comptes numériques (Richards, 1986).

En vue de dégager et de rehausser les structures linéaires correspondant à des discontinuités lithologiques ou structurales, un filtrage passe bas des opérateurs de type Sobel a été effectué. Il s'agit de filtres, de matrice 7 $\times 7$ (Figure 2), sélectionnés à partir des tests de Yésou et al. (1993) qui accentuent les directions lithologiques et structurales de la zone d'étude selon les quatre directions suivantes : E-O, N-S, NE-SO et NO-SE. En effet, la matrice constituant le filtre, de par sa nature même, entraîne le gommage ou l'estompage systématique des structures parallèles à la direction du filtre et au contraire le ravivage de celles qui lui sont orthogonales (Biémi, 1992 ; Kouamé, 1999 ; Jourda, 2005). 


$$
\left(\begin{array}{lllllll}
0 & 1 & 1 & 1 & 1 & 1 & 2 \\
-1 & 0 & 2 & 2 & 2 & 3 & 1 \\
-1 & -2 & 0 & 3 & 4 & 2 & 1 \\
-1 & -2 & -3 & 0 & 3 & 2 & 1 \\
-1 & -2 & -4 & -3 & 0 & 2 & 1 \\
-1 & -3 & -2 & -2 & -2 & 0 & 1 \\
-2 & -1 & -1 & -1 & -1 & -1 & 0
\end{array}\right)
$$

Filtre N-S

$$
\left(\begin{array}{lllllll}
2 & 1 & 1 & 1 & 1 & 1 & 0 \\
1 & 3 & 2 & 2 & 2 & 0 & 1 \\
1 & 2 & 4 & 3 & 0 & -2 & -1 \\
1 & 2 & 3 & 0 & -3 & -2 & -1 \\
1 & 2 & 0 & -3 & -4 & -2 & -1 \\
1 & 0 & -2 & -2 & -2 & -3 & -1 \\
0 & -1 & -1 & -1 & -1 & -1 & -2
\end{array}\right)
$$

Filtre E-O

$$
\left(\begin{array}{lllllll}
1 & 1 & 1 & 2 & 1 & 1 & 1 \\
1 & 1 & 2 & 3 & 2 & 1 & 1 \\
1 & 2 & 3 & 4 & 3 & 2 & 1 \\
0 & 0 & 0 & 0 & 0 & 0 & 0 \\
-1 & -2 & -3 & -4 & -3 & -2 & -1 \\
-1 & -1 & -2 & -3 & -2 & -1 & -1 \\
-1 & -1 & -1 & -2 & -1 & -1 & -1
\end{array}\right)
$$

Filtre NE-SO

$$
\left(\begin{array}{lllllll}
-1 & -1 & -1 & 0 & 1 & 1 & 1 \\
-1 & -1 & -2 & 0 & 2 & 1 & 1 \\
-1 & -2 & -3 & 0 & 3 & 2 & 1 \\
-2 & -3 & -4 & 0 & 4 & 3 & 2 \\
-1 & -2 & -3 & 0 & 3 & 2 & 1 \\
-1 & -1 & -2 & 0 & 2 & 1 & 1 \\
-1 & -1 & -1 & 0 & 1 & 1 & 1
\end{array}\right)
$$

Filtre NO-SE

Figure 2 : Matrices des filtres appliqués.

L’extraction manuelle des linéaments à l'écran a été réalisée sur les compositions colorées des ratios de canaux TM3/TM4, TM4/TM6, TM4/TM7 ainsi que sur les néocanaux issus du filtrage directionnel. Elle permet donc de produire des fichiers vectoriels issus des néo-canaux de l'analyse texturale (Youan Ta, 2008 ; Youan Ta et al., 2008 ; N'go et al., 2010). Les traitements numériques ont été effectués sur les canaux TM4, 5 et 7 qui ont été sélectionnés pour leurs caractéristiques spectrales et spatiales (30 m) permettant une analyse structurale ainsi qu'une cartographie à grande échelle (Youan Ta, 2008 ; Youan Ta et al., 2008). Un tri permet d'éliminer les artéfacts correspondant aux infrastructures. Les linéaments liés aux activités anthropiques (routes, chemin de fer, lignes de transport d'énergie, layons de délimitation des forêts, etc.) ont été supprimés de l'ensemble des éléments structuraux. La carte linéamentaire obtenue est celle ayant trait à la tectonique.

\section{RESULTATS \\ Hydrochimie \\ Paramètres physico-chimiques}

Les valeurs moyennes des paramètres physico-chimiques mesurés sont indiquées dans le Tableau 1. Les eaux de surface sont peu minéralisées, relativement plus chaudes avec un $\mathrm{pH}$ proche de la neutralité. Les nappes de fissures plus profondes enregistrent une minéralisation moyenne avec un $\mathrm{pH}$ plus acide et une température plus faible. Les aquifères des altérites présentent la plus forte minéralisation au niveau des trois types d'échantillons d'eaux étudiés. L'acidité de ces eaux est légèrement inférieure à celle des fissures et supérieure à celle des eaux de surface. Il en est de même pour la température.

Les conductivités des différents types d'échantillons (eaux de surface, des altérites et des fissures) varient respectivement entre 40 et $110 \mu \mathrm{S} / \mathrm{cm}, 250$ et $1690 \mu \mathrm{S} / \mathrm{cm}$ et 30 et $1400 \mu \mathrm{S} / \mathrm{cm}$. En dehors des eaux de surface dont la conductivité électrique varie très peu pour des récurrences fortement variables, les 
eaux souterraines (altérites et fissures) présentent une récurrence de plus en plus faible pour des C.E. de plus en plus élevées. La minéralisation totale des eaux des aquifères de fissures s'intègrent entre celle des eaux de surface et des aquifères des altérites avec un nombre important d'échantillons se comportant comme des eaux de surface (pour des C.E. $<200 \mu \mathrm{S} / \mathrm{cm}$, Figure 3). Notons que pour des conductivités électriques supérieures à $200 \mu \mathrm{S} / \mathrm{cm}$ les eaux des aquifères de fissures se rapprochent plus des eaux de surface que des eaux des altérites.

\section{Eléments chimiques dissous}

Les eaux de surface sont moins minéralisées que les eaux des aquifères fissurés lesquelles sont moins minéralisées que les eaux des altérites (Figure 4). $\mathrm{Au}$ niveau de ces trois types d'échantillons, les teneurs moyennes en silice $\left(\mathrm{SiO}_{2}\right)$, phosphate $\left(\mathrm{PO}_{4}{ }^{3-}\right)$ et nitrites $\left(\mathrm{NO}_{2}^{-}\right)$sont comparables. L'analyse des autres éléments chimiques indique que les plus fortes teneurs sont enregistrées au niveau des aquifères des altérites, suivies des aquifères fissurés. Le calcium $\left(\mathrm{Ca}^{2+}\right)$ et le sodium $\left(\mathrm{Na}^{+}\right)$apparaissent comme les cations dominants pour l'ensemble des eaux souterraines (aquifères de fissures et des altérites). Les bicarbonates $\left(\mathrm{HCO}_{3}{ }^{-}\right)$et les chlorures $\left(\mathrm{Cl}^{-}\right)$représentent les anions dominants pour la quasi-totalité des eaux échantillonnées au niveau de la zone d'étude.

\section{Hydrofaciès}

L'analyse des eaux dans le diagramme de Piper permet d'observer deux pôles hydrochimiques majeurs : bicarbonaté calcomagnésien et chloruré nitraté calco-magnésien (Figure 5). Le faciès bicarbonaté calco- magnésien apparaît le plus dominant. On l'observe dans la quasi-totalité des points d'eaux de surface, au niveau de $70 \%$ des eaux des aquifères fissurés et seulement $20 \%$ des eaux des aquifères d'altérites. Ce faciès apparaît être caractéristique d'une minéralisation naturelle car le bicarbonate, le calcium et le magnésium sont des produits de l'altération des matrices rocheuses encaissantes. Le faciès chloruré nitraté calcomagnésien est caractéristique d'une minéralisation induite (pollution au chlorure ou nitrate). Ce faciès représente respectivement $1 \%$ des eaux de surface, $23 \%$ des eaux des fissures et $36 \%$ des eaux des altérites. D’autres faciès moins important, bicarbinaté sodi-potassique et chloruré nitraté sodi-potassique,se rencontrent aussi dans la région.

\section{Télédétection : réseau de fractures du socle cristallin}

La carte du réseau linéamentaire (Figure 6) a été obtenue après les différents traitements des images satellitaires et le tri ayant permis d'éliminer les artéfacts correspondant aux infrastructures de développement.

Les objets linéaires résultants sont assimilés au réseau de fractures et sont utilisés pour la relation du réseau de linéaments de surface avec les directions majeures d'écoulement souterrain en milieu cristallin. Il existe une superposition parfaite entre les directions tectoniques majeures relevées sur les images satellitaires et la répartition spatiale de l'ensemble des drains formant le réseau hydrographique au sol (Figure 7).

Tableau 1 : Valeurs moyennes des paramètres physico-chimiques mesurés dans les eaux de surface, les nappes d'altérites et les nappes de fissures.

\begin{tabular}{lccc}
\hline Hydrosystèmes & $\mathbf{T}\left({ }^{\circ} \mathbf{C}\right)$ & $\mathbf{p H}$ & $\mathbf{C . E .}(\boldsymbol{\mu S} / \mathbf{c m})$ \\
\hline Eau de surface & $31,0 \pm 13,0$ & $7,5 \pm 0,7$ & $80,4 \pm 20,6$ \\
Nappe d'altérites & $27,0 \pm 2,8$ & $6,9 \pm 0,9$ & $953,0 \pm 524,6$ \\
Nappe de fissures & $26,5 \pm 2,1$ & $6,5 \pm 0,6$ & $447,8 \pm 80,4$ \\
\hline
\end{tabular}




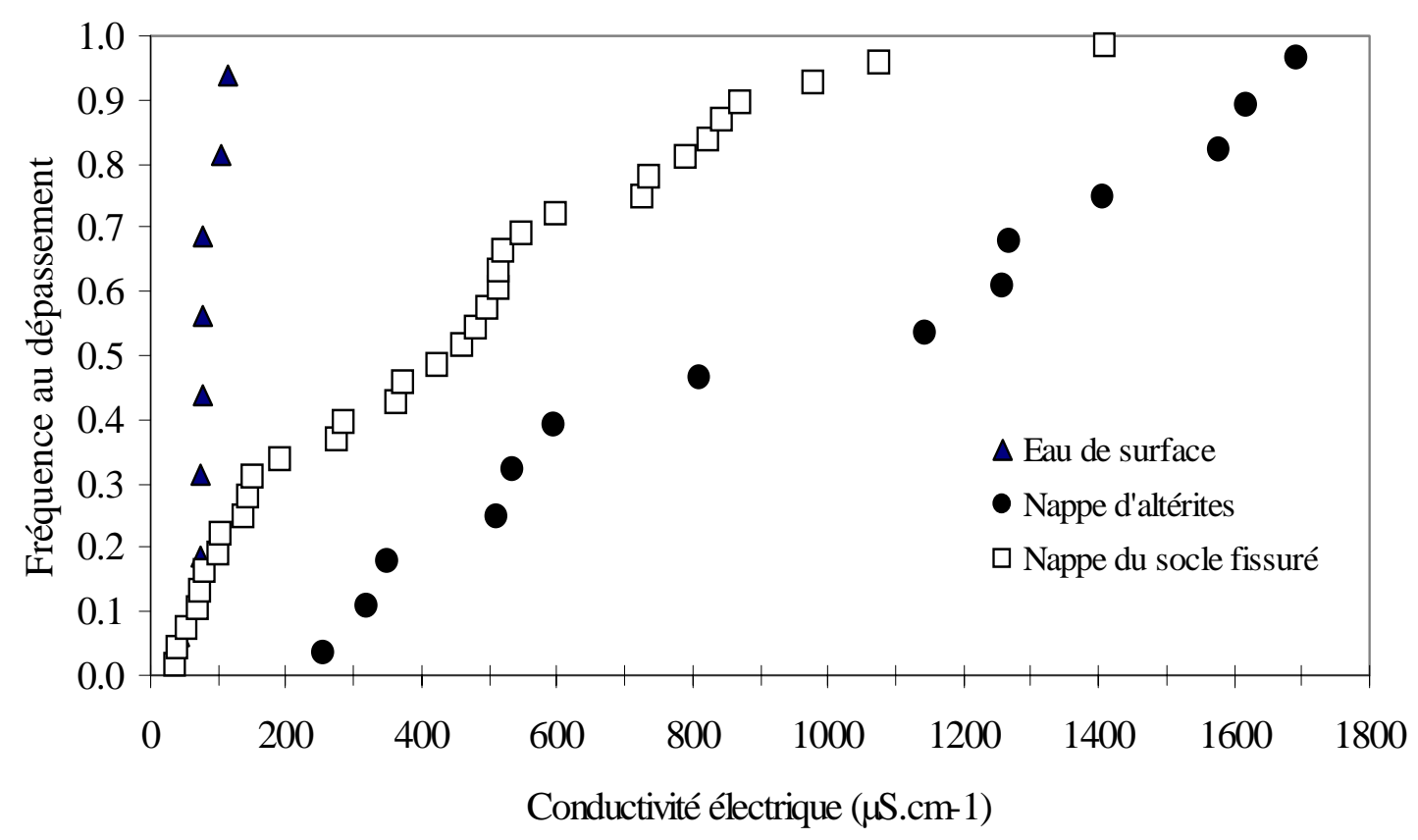

Figure 3 : Fréquence d’apparition des conductivités électriques des eaux souterraines et de surface.

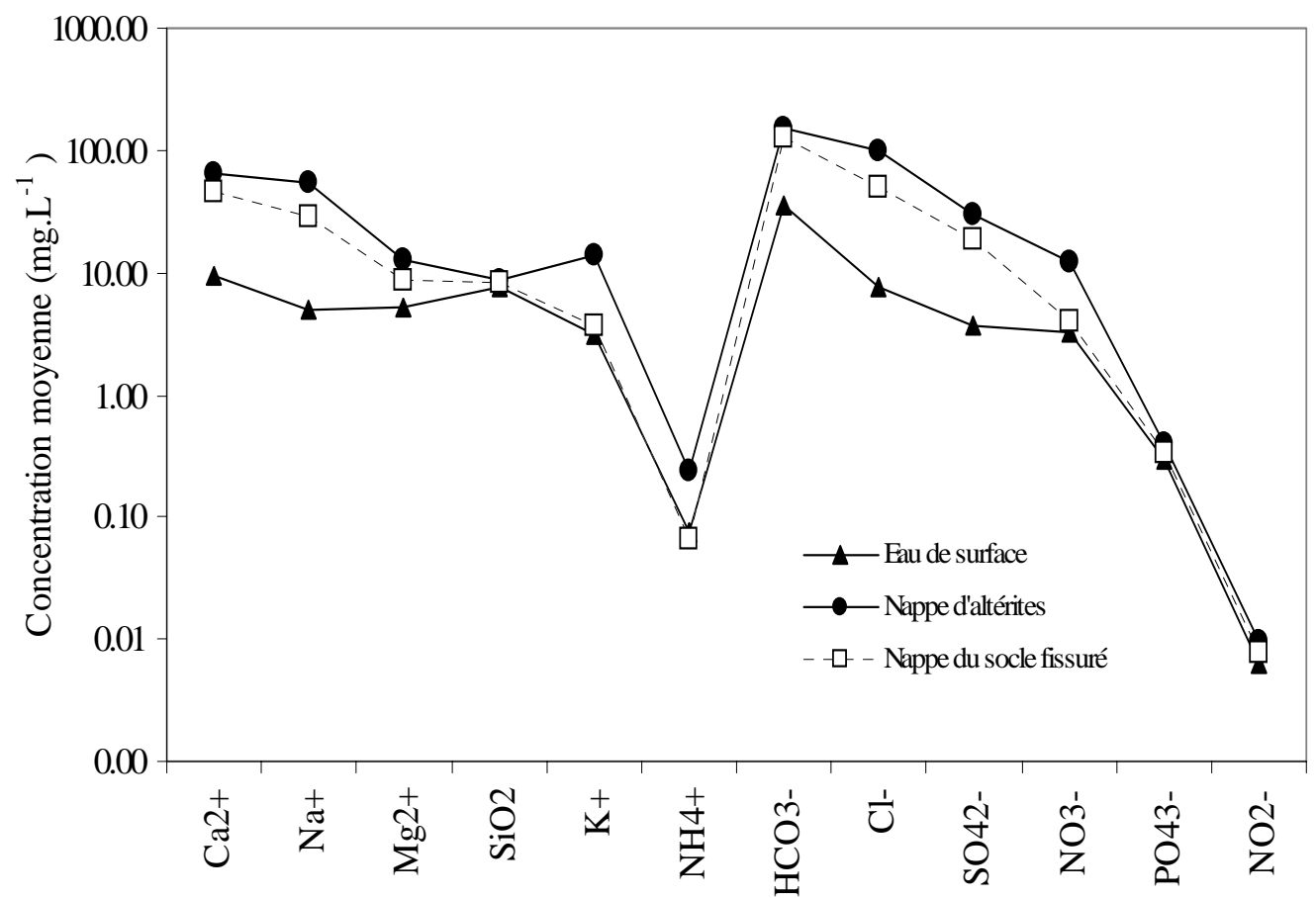

Figure 4 : Composition chimique moyenne des eaux de surface, et des nappes d'altérites et de socle. 


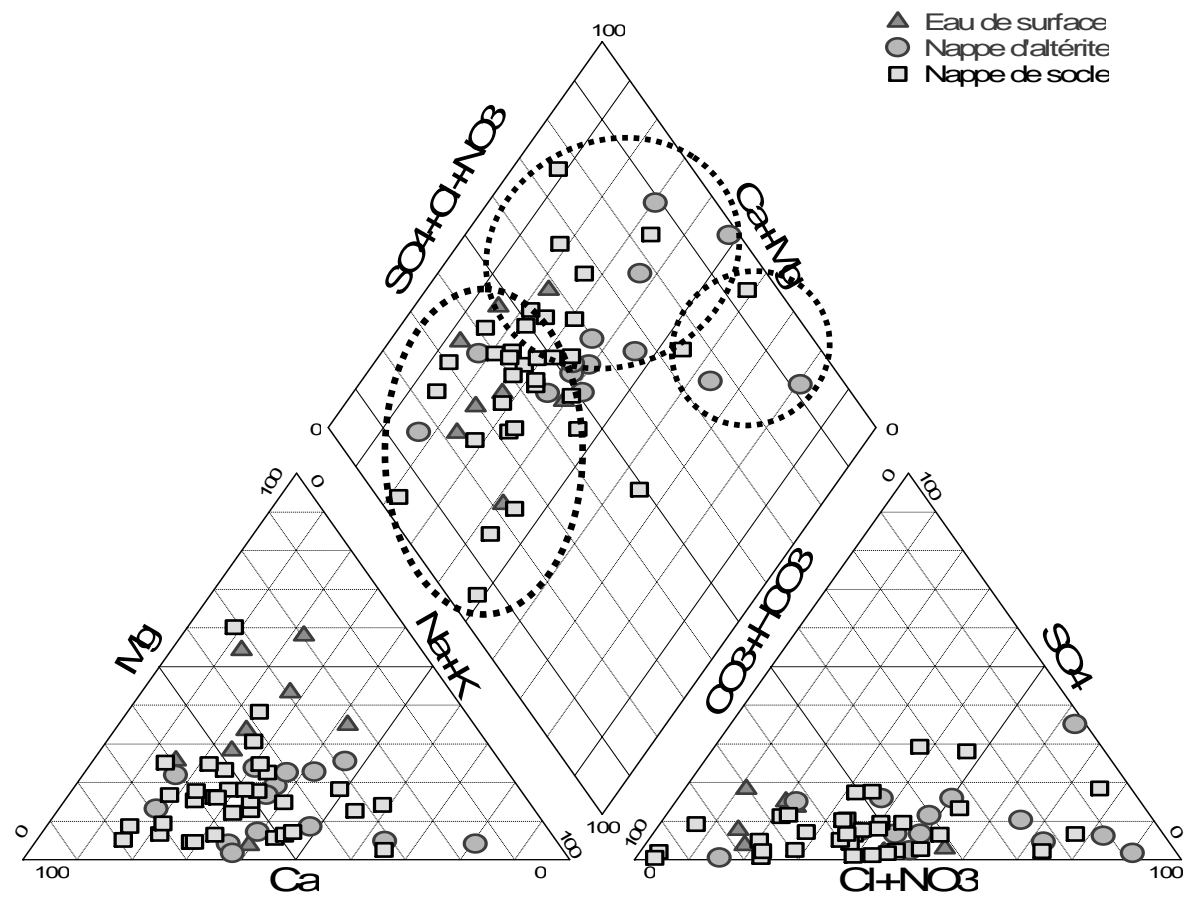

Figure 5 : Diagramme de Piper des hydrosystèmes étudiés.

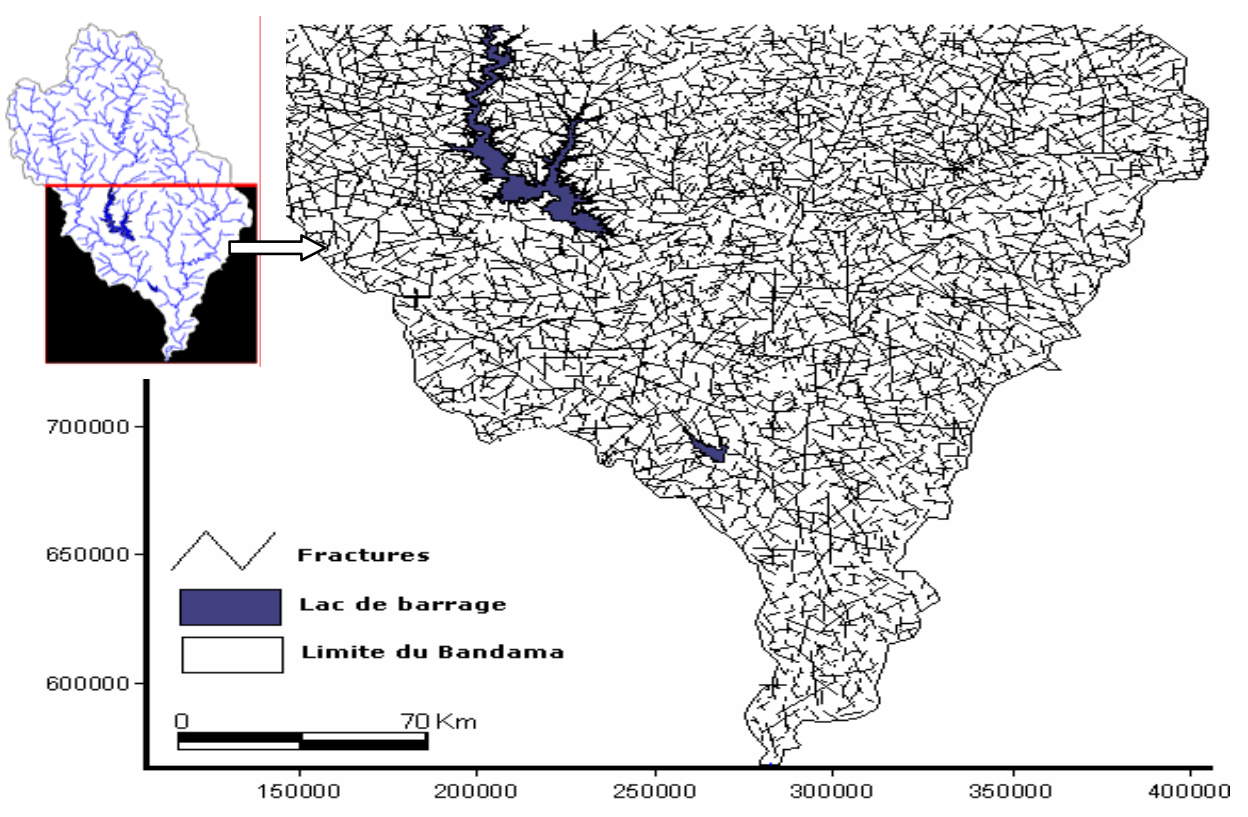

Figure 6 : Carte linéamentaire détaillée. 


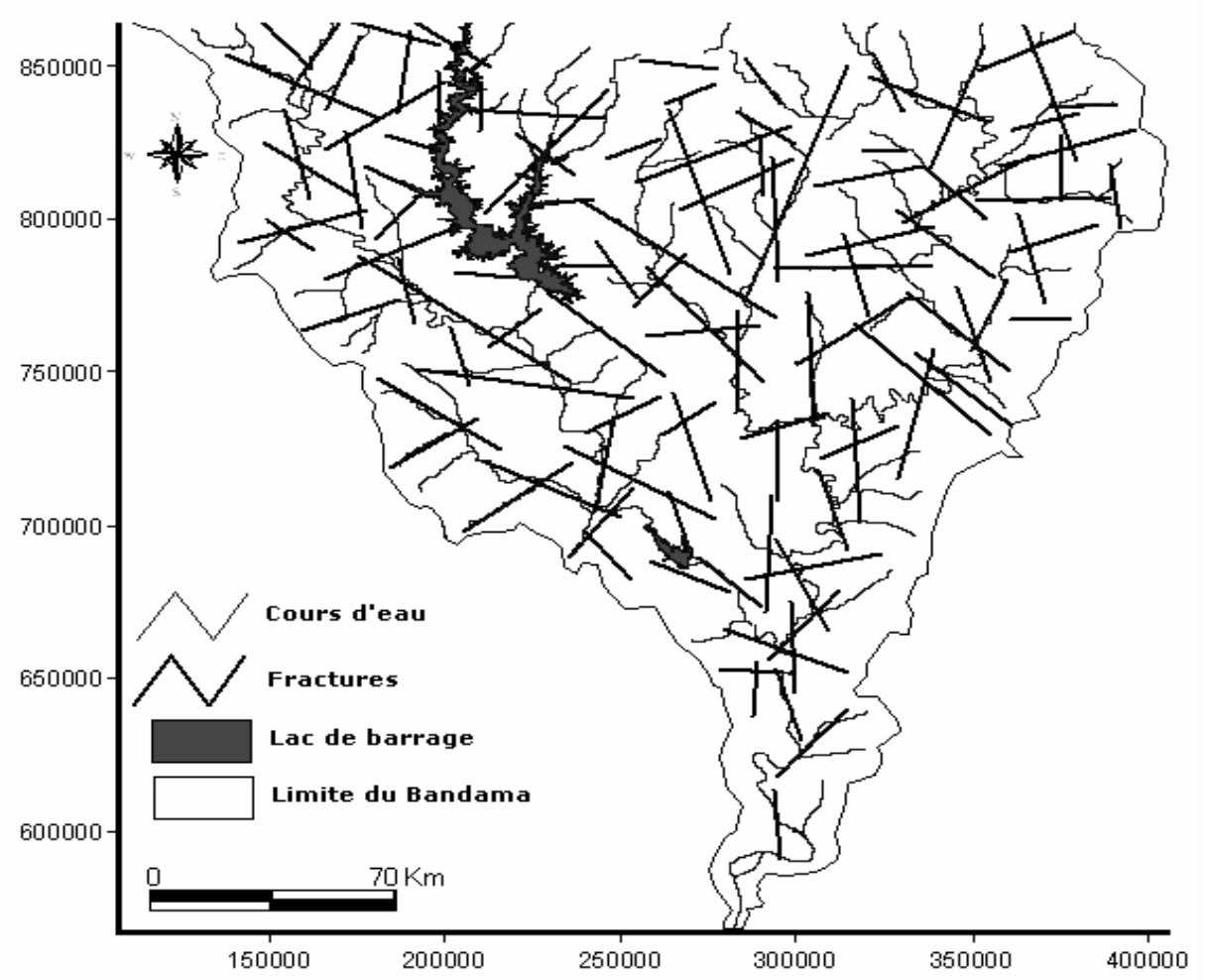

Figure 7 : Superposition du réseau hydrographique au réseau de fractures majeures du socle fissuré.

\section{DISCUSSION}

De façon générale, les eaux étudiées présentent une minéralisation relativement faible. Ceci est à relier à la nature silicatée des matrices rocheuses traversées dont l'altération et la mise en solution des éléments chimiques sous forme ionique sont particulièrement lentes.

Les eaux de surface présentent logiquement une minéralisation plus faible que celle de l'ensemble des eaux souterraines. Ceci est imputable à son caractère de système ouvert à l'atmosphère qui reçoit directement les précipitations faiblement minéralisées. Cependant, les eaux des aquifères d'altérites indiquent paradoxalement des conductivités électriques plus fortes que celles des aquifères fissurés. Des études réalisées en milieu de socle cristallin en Côte d'Ivoire (Savané, 1997 ; Goné, 2001) et au Bénin (Kamagaté, 2006) révèlent que les eaux des nappes de fissures, plus profondes, seraient les plus riches en sels dissous du fait d'un temps de résidence et une distance de transit plus longs pour les eaux de recharge. Cette interprétation semble peu probable ici puisque ce sont les eaux des altérites qui enregistrent les plus fortes minéralisations. Cette minéralisation dans les altérites pourrait être liée, outre aux processus naturels de minéralisation (hydrolyse des silicates notamment), à des phénomènes de pollution aux nitrates et chlorures (Faillat et Drogue, 1993) dus probablement à la forte anthropisation du bassin (utilisation d'engrais et pesticides, effluents des ouvrages d'assainissement). Une faible connexion entre ces deux aquifères qui ralentirait la continuité hydraulique et donc un mélange de ces deux types d'eaux peut également être évoquée.

Les eaux des aquifères de fissures ont une signature chimique proche des eaux de surface traduisant une interaction possible entre ces deux hydrosystèmes. Cette assertion 
est confortée par la superposition à plusieurs endroits des réseaux hydrographique et de fractures du socle constituant l'aquifère de fissures. En l'absence de niveau piézométrique en continue et de nivellement de l'ensemble des points de mesures, deux hypothèses peuvent être évoquées en relation avec le transfert de flux et l'échange d'eau entre réseau hydrographique et réseau de fracture du socle : i) les cours d'eau pourraient constituer des drains de vidange pour les aquifères du socle fissuré, ii) les fractures pourraient constituer des couloirs permettant l'évacuation de la partie immergée (invisible) des eaux de surface (Savané et al., 1993). Cette deuxième approche paraît plausible, ces réservoirs de fissures seraient situés dans des substratums à couloirs mylonitiques où les structures conductrices sont bien développées. Ceci est en accord avec la présence de failles superficielles et récentes repérées lors de la fondation du barrage de Taabo (Kaiser Engineers et Constructors, 1980) situé à l'amont de la zone étudiée.

\section{Conclusion}

L'objectif de ce travail était de dégager le type de connexion entre le réseau hydrographique et les eaux souterraines à partir d'une approche couplée hydrochimie et télédétection.

Du point de vue hydrochimique, les eaux de surface présentent une signature chimique proche de celle des nappes du socle fissuré. Les aquifères des altérites, moins profonds, semblent être faiblement connectés aux deux premiers systèmes avec un faible échange d'eau. Ceci milite en faveur d'un échange de flux entre le réseau hydrographique et les aquifères fissurés, sans contribution significative des aquifères d'altérites. Du point de vue de la télédétection, cette interprétation est en accord avec la parfaite superposition entre le réseau des cours d'eau et le réseau des fractures du socle cristallin.

On pourrait donc retenir que dans le bassin versant du Bandama, les fractures du socle cristallin draine une partie de l'eau contenue dans le réseau hydrographique.

\section{REFERENCES}

Biémi J. 1992. Contribution à l'étude géologique, hydrogéologique et par télédétection des bassins versants subsaheliens du socle précambrien de l'Afrique de l'Ouest: Hydrostructurale, hydrodynamique, hydrochimie et isotopie des aquifères discontinus de sillons et aires granitiques de la haute Marahoué (Côte d'Ivoire). Thèse de doctorat d'Etat de l'Université d'Abidjan, Côte d'Ivoire, p. 493.

Braun JJ, Dupré B, Viers J, Ndam Ngoupayou JR, Bedimo Bedimo JP, Sigha-Nkamdjou L, Freydier R, Robain H, Nyeck B, Bodin J, Oliva P, Boeglin JL, Stemmler S, Berthelin J. 2002. Biogeohydrodynamic in the forested humid tropical environment: the case study of the Nsimi small experimental watershed (south Cameroon). Bull. Soc. Geol. France, 173: 347-357.

Dao A, Kamagaté B, Mariko A, Goula Bi TA, Seguis L, Maïga HB, Savane I. 2010. Variabilité Climatique et Réponse Hydrologique du Bassin Versant Transfrontalier de Kolondièba au Sud du Mali. Euro. J. Sci. Resear., 43(4): 435444.

Doumouya I, Kamagaté B, Bamba A, Ouedraogo M, Ouattara I, Savane I, Goula Bi TA, Biémi J. 2009. Impact de la variabilité climatique sur les ressources en eau et végétation du bassin versant du Bandama en milieu intertropical (Côte d'Ivoire). Rev. Ivoir. Sci. Technol., 14: 203-215.

Faillat JP, Drogue C. 1993. Différenciation hydrochimique de nappes superposées d'altérites et de fissures en socle granitique. J. Sci. Hydrol., 38(3): 215229.

Goné DL. 2001. Contribution des paramètres physico-chimiques des eaux souterraines 
à l'étude du fonctionnement des systèmes hydrauliques en milieu fissuré de la région sémi-montagneuse de Man (Ouest de la Côte d'Ivoire). Thèse de Doctorat de l’Université d'Abobo-Adjamé, Côte d'Ivoire, p. 182.

Goula BTA, Savané I, Konan B, Fadika V, Kouadio GB. 2006. Impact de la variabilité climatique sur les ressources hydriques des bassins de N'zo et N'zi en Côte d'Ivoire (Afrique tropicale humide). VertigO, Rev. Ssi. Evironment, 7(1): 12.

Grillot JC. 1992. Régime des eaux souterraines en milieu cristallin altéré : un exemple en zone intertropicale humide d'altitude (Madagascar). J. Sci. Hydrol., 37(2): 105-117.

Jourda JPR. 2002. Les ressources en eau souterraine de Côte d'Ivoire et le cas des aquifères transfrontaliers entre la Côte d'Ivoire et le Ghana. Managing shared aquifer resources in Africa UNESCO, Paris, France, 87-92.

Jourda JP. 2005. Méthodologie d'application des techniques de télédétection et des systèmes d'information géographique à l'étude des aquifères fissurés d'Afrique de l'ouest. Concept de l'Hydrotechnique spatiale : cas des zones tests de la Côte d'Ivoire. Thèse de doctorat de l'Université de Cocody, Côte d'Ivoire, p. 429.

Kaiser Engineers and Constructors, INCUSA. 1980. Projet hydroélectrique de Taabo. Rapport final de l'aménagement, AFFAIRE N73118, vol. 2, p. 263.

Kamagaté B. 2006. Fonctionnement Hydrologique et origines des écoulements sur un bassin versant de milieu tropical de socle au Bénin : Bassin versant de Donga (haute vallée de l’Ouémé). Thèse de Doctorat de l'Université de Montpellier II, France, p. 319.

Kouamé KF. 1999. Hydrogéologie des aquifères discontinus de la région semi-montagneuse de Man-Danané (Ouest de la Côte d'Ivoire). Apport des données des images satellitales et des méthodes statistique et fractale à l'élaboration d'un système d'information hydrogéologique à référence spatiale. Thèse de doctorat de l'Université de Cocody, Côte d'Ivoire, p. 210.

Lebel T, Vischel T. 2005. Climat et cycle de l'eau en zone tropicale : un problème d'échelle. C. R. Geosci., 337: 29-38.

Monnet C. 1970. Transport solide en suspension par un fleuve de Côte d'Ivoire: le Bandama. Rapport de L'ORSTOM, centre d'Adopodoumé Côte d'Ivoire, Laboratoire de sédimentologie, p. 157.

N’Go AY, Lasm T, Koïta M, Savane I. 2010. Extraction par télédétection des réseaux de fractures majeures du socle précambrien de la région de Dimbokro (Centre-Est de la Côte d'Ivoire). Télédétection, 9(1): 33-42.

Ouattara I. 2009. Processus hydrogéochimiques en milieu soudano-tropical de socle cristallin en Côte d'Ivoire: Bassin versant du Bandama. DEA de l’Université d'Abobo-Adjamé, Côte d'Ivoire, p. 79.

Ouédraogo M. 2009. Caractérisation des états de surface et impact des changements de l'occupation du sol sur les ressources en eau dans le bassin versant du Bandama. DEA de l'Université d'Abobo-Adjamé, Côte d'Ivoire, p. 74.

Richards JA. 1986. Feature Reduction in Remote Sensing Digital Image Analysis. An introduction. Springer-Verlag: Berlin ; 281.

Savané I, Bénié GB, Gwyn QJH, Biémi J. 1993. Application de la télédétection à la recherche des eaux souterraines en milieu cristallin : Cas d’Odienné, Côte d’Ivoire. Actualité scientifique, AUPELF-AUREF. Télédétection des ressources en eau. Actes des journées scientifiques de Tunis, 111-120.

Savané I. 1997. Contribution à l'étude géologique et hydrogéologique des 
aquifères discontinus du socle cristallin d’Odienné (Nord-Ouest de la Côte d'Ivoire). Apport de la Télédétection et d'un système d'information hydrogéologique à référence spatiale. Thèse de Doctorat de l'Université de Cocody, Abidjan, p. 396.

Servat E, Paturel JE, Lubes-Niel H, Kouame B, Masson JM, Travaglio M. 1997. Climatic variability in humid Africa along the Gulf of Guinea - Part one: detailed analysis of the phenomenon in Côte d'Ivoire. J. Hydrol., 191: 7-15.

Yao KA, Yao GF, Alui AK, N’Guessan AK, Tiémoko TP, Yao Konan Kloman YK. 2008. Etude morphopédogique du bassin versant du mont Blanguand dans le massif du Yaouré en région centre de la Côte d'Ivoire. Afrique Sci., 4(3): 426-451.

Yésou H, Saint-Jean R, Pion JC, Besnus R. 1993. Amélioration des données SPOT pour la cartographie structurale en milieu tropical. Exemple de la région des chapeaux de fer de Pagala (Togo). p. 143164. In Outils Micro-Informatiques et Télédétection de l'Evolution des Milieux, Dubois J-MM, Blasco F (eds). Série Actualité scientifique, Presses de l'Université du Québec/AUPELF: SainteFoy ; 492.

Youan TM, Lasm T, Jourda JP, Kouame KF, Razack M. 2008. Cartographie structurale par imagerie satellitaire ETM+ de Landsat-7 et analyse des réseaux de fractures du socle précambrien de la région de Bondoukou (nord-est de la cote d'Ivoire). Télédétection, 2: 34-42.

Youan TM. 2008. Contribution de la télédétection et des systèmes d'informations géographiques à la prospection hydrogéologique du socle précambrien d'Afrique de l'Ouest: Cas de la région de Bondoukou Nord Est de la Côte d'Ivoire. Thèse de doctorat de l'Université de Cocody, Côte d'Ivoire, p. 236. 